\title{
CONSTRUCTING HERMAN RINGS BY TWISTING ANNULUS HOMEOMORPHISMS
}

\author{
XIUMEI WANG and GAOFEI ZHANG ${ }^{凶}$ \\ (Received 23 December 2006; accepted 25 March 2007) \\ Communicated by P. C. Fenton
}

\begin{abstract}
Let $F(z)$ be a rational map with degree at least three. Suppose that there exists an annulus $H \subset \widehat{\mathbb{C}}$ such that (1) $H$ separates two critical points of $F$, and (2) $F: H \rightarrow F(H)$ is a homeomorphism. Our goal in this paper is to show how to construct a rational map $G$ by twisting $F$ on $H$ such that $G$ has the same degree as $F$ and, moreover, $G$ has a Herman ring with any given Diophantine type rotation number.
\end{abstract}

2000 Mathematics subject classification: primary 30D05; secondary 37F10, 37F45, 32H50.

Keywords and phrases: Herman rings.

\section{Introduction}

Let $f$ be a rational map with degree not less than two. Let $H$ be an invariant Fatou component of $f$. We say that $H$ is a Herman ring if $f: H \rightarrow H$ is holomorphically conjugate to an irrational rotation of some annulus. There are two known methods to construct a Herman ring. The original method, which is due to Herman, is based on certain Blaschke products and Arnold's linearization theorem on real-analytic circle homeomorphisms. A more general construction was proposed by Shishikura. The idea of Shishikura can be sketched as follows. One starts with two rational maps with Siegel disks of rotation number $\theta$ and $-\theta$. In order to fabricate a Herman ring, one needs to cut and paste together the two Siegel disks to get a topological model map. Then by using the Morrey-Ahlfors-Bers Measurable Riemann Mapping theorem, one can conjugate the resulted topological picture to an actual rational map. For more details about these two constructions, the reader may refer to [1, Ch. VI].

In this paper, we extend Herman's idea and provide an another way to construct Herman rings by twisting a rational map on some annulus. Here the word twisting means, for any given Diophantine type irrational number $0<\theta<1$, we

The second author is partially supported by NJU-0203005116.

(c) 2009 Australian Mathematical Society 1446-7887/2009 \$16.00 
can postcompose the rational map by a certain homeomorphism such that the resulting model map, when restricted to some topological annulus, is quasiconformally conjugate to the rigid rotation $z \rightarrow e^{2 \pi i \theta} z$. This idea comes from [4] where a Siegel disk is constructed by twisting a linearizable domain centered at an attracting fixed point. Compared with the constructions given by Herman and Shishikura, the new feature of our construction is that the starting point is just a rational map which satisfies a fairly general topological condition. Before we state the Main Theorem, let us first introduce a definition.

Definition 1.1. Let $F$ and $G$ be two rational maps. We say that $F$ and $G$ are topologically equivalent to each other if there exist homeomorphisms $\phi, \psi: \widehat{\mathbb{C}} \rightarrow \widehat{\mathbb{C}}$ such that $F \circ \phi=\psi \circ G$.

Recall that an irrational number $0<\theta<1$ is called a Diophantine number if there exist $\beta \geq 2$ and $C>0$ such that for all positive integers $p$ and $q,|\theta-p / q|>C / q^{\beta}$. We prove the following result.

MAIN TheOREM. Let $0<\theta<1$ be a Diophantine number and $F(z)$ be a rational map with degree at least three. Suppose that there exists an annulus $H \subset \widehat{\mathbb{C}}$ such that (1) $H$ separates two critical points of $F$, and (2) $F: H \rightarrow F(H)$ is a homeomorphism. Then there exists a rational map $G$ which has a Herman ring with rotation number $\theta$ such that $F$ and $G$ are topologically equivalent to each other.

REMARK 1.2. The Main Theorem applies for a slightly larger class of rotation numbers which are called Herman numbers. This is because the arithmetic condition of $\theta$ is only needed to apply the following Herman-Yoccoz Theorem and this theorem is actually true for all such rotation numbers (the reader may refer to [3, p. 131] for the definition of Herman numbers).

Let $R_{\theta}$ denote the rigid rotation given by $z \rightarrow e^{2 \pi i \theta} z$.

TheOREM (HeRMAN-YocCOZ) [3]. Let $0<\theta<1$ be a Diophantine number. Let $f: S^{1} \rightarrow S^{1}$ be a real-analytic circle diffeomorphism of rotation number $\theta$. Then $f$ is real-analytically conjugate to the rigid rotation $R_{\theta}: S^{1} \rightarrow S^{1}$.

REMARK 1.3. The condition that the degree of $F$ is not less than three is forced by the topological restriction: for a quadratic rational map $F$, there is no annulus which separates the two critical points, and on which $F$ is a homeomorphism.

\section{Proof of the Main Theorem}

Let $F$ and $H$ be the rational map and the annulus which satisfy the conditions in the Main Theorem. Without loss of generality, we may assume that the two critical points separated by $H$ are 0 and $\infty$. 
2.1. The Riemann isomorphisms $\boldsymbol{g}_{\xi}$ and $\boldsymbol{h}_{\lambda}$ By considering a sub-annulus of $H$, we may assume that both boundaries of $H$ are real-analytic curves which do not pass any critical point of $F$. Let $\gamma$ and $\eta$ denote the outer and the inner boundary component of $H$, respectively. It follows that the curves $F(\gamma)$ and $F(\eta)$, which are the two boundary components of $F(H)$, are also real-analytic curves.

Let $U$ and $V$ denote the two components of $\widehat{\mathbb{C}}-\bar{H}$ such that $\partial U=\gamma$ and $\partial V=\eta$. By the assumption, we obtain $\infty \in U$ and $0 \in V$. Let $X$ and $Y$ denote the two components of $\widehat{\mathbb{C}}-\overline{F(H)}$ such that $\partial X=F(\gamma)$ and $\partial Y=F(\eta)$. Take $x \in X, y \in Y$, $a \in \partial X$, and $b \in \partial Y$.

For each $\xi \in \gamma$, there is a unique holomorphic isomorphism $g_{\xi}: X \rightarrow U$ such that $g_{\xi}(a)=\xi$ and $g_{\xi}(x)=\infty$. The maps $g_{\xi} \circ F \mid \gamma: \gamma \rightarrow \gamma, \xi \in \gamma$, consist of a monotone family of topological circle homeomorphisms. By [2, Proposition 11.1.9], it follows that there is a unique $\xi^{\prime} \in \gamma$ such that the rotation number of $g_{\xi^{\prime}} \circ F \mid \gamma: \gamma \rightarrow \gamma$ is $\theta$.

Similarly, for each $\lambda \in \eta$, there is a unique holomorphic isomorphism $h_{\lambda}: Y \rightarrow V$ such that $h_{\lambda}(b)=\lambda$ and $h_{\lambda}(y)=0$. The maps $h_{\lambda} \circ F: \eta \rightarrow \eta$, where $\lambda \in \eta$, consist of a monotone family of topological circle homeomorphisms. Again by [2, Proposition 11.1.9], it follows that there is a unique $\lambda^{\prime} \in \eta$ such that the rotation number of $h_{\lambda^{\prime}} \circ F \mid \eta: \eta \rightarrow \eta$ is $\theta$.

2.2. Passing to an Euclidean annulus Take $0<r<R$. Let $\Delta_{R}$ and $\Delta_{r}$ denote the two Euclidean disks which are centered at the origin, and which have radius $R$ and $r$, respectively. Let $\mathbb{T}_{R}=\partial \Delta_{R}$ and $\mathbb{T}_{r}=\partial \Delta_{r}$. Let $\phi: \widehat{\mathbb{C}}-\overline{\Delta_{R}} \rightarrow U$ be the Riemann isomorphism such that $\phi(\infty)=\infty$ and $\phi^{\prime}(\infty)>0$. Similarly, let $\psi: \Delta_{r} \rightarrow V$ be the Riemann isomorphism such that $\psi(0)=0$ and $\psi^{\prime}(0)>0$. Since $\xi$ and $\eta$ are both realanalytic curves, it follows that $\phi$ and $\psi$ can be homeomorphically extended to $\mathbb{T}_{R}$ and $\mathbb{T}_{r}$, respectively. By the Schwarz Reflection Lemma, one easily obtains the following result.

LEMMA 2.1. Both the maps $\phi^{-1} \circ g_{\xi^{\prime}} \circ F \circ \phi: \mathbb{T}_{R} \rightarrow \mathbb{T}_{R}$ and $\psi^{-1} \circ h_{\lambda^{\prime}} \circ F \circ \psi$ : $\mathbb{T}_{r} \rightarrow \mathbb{T}_{r}$ are real-analytic circle diffeomorphisms of rotation number $\theta$.

Now applying the Herman-Yoccoz Theorem (see Section 1), we obtain the following result.

LEMMA 2.2. There exist two analytic circle homeomorphisms $h_{1}: \mathbb{T}_{R} \rightarrow \mathbb{T}_{R}$ and $h_{2}: \mathbb{T}_{r} \rightarrow \mathbb{T}_{r}$ such that $\phi^{-1} \circ g_{\xi^{\prime}} \circ F \circ \phi(z)=h_{1}^{-1} \circ R_{\theta} \circ h_{1}(z)$ for all $z \in \mathbb{T}_{R}$, and $\psi^{-1} \circ h_{\lambda^{\prime}} \circ F \circ \psi(z)=h_{2}^{-1} \circ R_{\theta} \circ h_{2}(z)$ for all $z \in \mathbb{T}_{r}$.

Let $A$ denote the annulus $\{z|r<| z \mid<R\}$. Since $h_{1}: \mathbb{T}_{R} \rightarrow \mathbb{T}_{R}$ and $h_{2}: \mathbb{T}_{r} \rightarrow \mathbb{T}_{r}$ are both real-analytic homeomorphisms, it is not difficult to obtain the following result.

LEMMA 2.3. There exists a quasiconformal homeomorphism $\sigma: A \rightarrow A$ such that $\sigma \mid \mathbb{T}_{R}=h_{1}$ and $\sigma \mid \mathbb{T}_{r}=h_{2}$.

Since $\gamma$ and $\eta$ are real-analytic curves, one can easily obtain the following result. 
LEMMA 2.4. There is a quasiconformal homeomorphism $\tau: A \rightarrow H$ such that $\tau \mid \mathbb{T}_{R}=\phi$ and $\tau \mid \mathbb{T}_{r}=\psi$.

2.3. The quasiconformal model map Since $F: H \rightarrow F(H)$ is a homeomorphism, there is an inverse branch of $F$ defined on $F(H)$, say $\chi: F(H) \rightarrow H$ such that $\chi \circ F=$ id on $H$. Since both $\eta$ and $\xi$ are analytic curves and are therefore quasiconformally erasable, we can define a quasi-conformal homeomorphism $L: \widehat{\mathbb{C}} \rightarrow \widehat{\mathbb{C}}$ by

$$
L(z)= \begin{cases}g_{\xi^{\prime}}(z) & \text { for all } z \in X, \\ \tau \circ \sigma^{-1} \circ R_{\theta} \circ \sigma \circ \tau^{-1} \circ \chi(z) & \text { for all } z \in F(\bar{H}), \\ h_{\lambda^{\prime}}(z) & \text { for all } z \in Y .\end{cases}
$$

Now define the quasiconformal model map $\widetilde{F}: \widehat{\mathbb{C}} \rightarrow \widehat{\mathbb{C}}$ by $\widetilde{F}(z)=L \circ F(z)$. Note that, by construction, $\widetilde{F}(z)$ is holomorphic on the outside of $\widetilde{F}^{-1}(\bar{H})$.

2.4. Realize the quasiconformal map $\tilde{\boldsymbol{F}}$ by a rational map By the construction of $\widetilde{F}$, it follows that when restricted on $H, \widetilde{F}$ is quasiconformally conjugate to the irrational rotation $R_{\theta}: A \rightarrow A$. Therefore, $\widetilde{F}$ has an invariant complex structure $\mu$ defined in $H$. Since $\widetilde{F}$ is holomorphic on the outside of $\widetilde{F}^{-1}(\bar{H})$, one can pull back $\mu$ by the iteration of $\widetilde{F}$ and finally obtain a $\widetilde{F}$-invariant complex structure $v$ on the whole Riemann sphere. Now applying the Morrey-Ahlfors-Bers Measurable Riemann Mapping Theorem, one has a quasiconformal homeomorphism $\omega$ of the sphere which fixes 0,1 , and $\infty$, and which solves the Beltrami equation given by $v$. Note that when restricted to $H, v=\mu$. This implies that the map $G(z)=\omega \circ \widetilde{F} \circ \omega^{-1}(z)$ is a rational map which, when restricted to $\omega(H)$, is holomorphically conjugated to the irrational rotation $R_{\theta}: A \rightarrow A$. Let $W$ be the Fatou component of $G$ which contains $\omega(H)$. It follows that $W$ is either a Siegel disk or a Herman ring. Since $\omega(H)$ separates 0 and $\infty$ which are critical points of $G$, by construction, it follows that $W$ cannot be simply connected. This implies that $W$ is a Herman ring of $G$ which has rotation number $\theta$. By the construction, it is clear that $G$ is topologically equivalent to $F$. The proof of the Main Theorem is complete.

REMARK 2.5. There are many ways to construct a rational map $F$ which satisfies the conditions in the Main Theorem. For instance, let $F(z)=z+\epsilon\left(z^{m}+1 / z^{n}\right)$ where $m, n \geq 2$ are integers. It is not difficult to see that when $|\epsilon|>0$ is small, there is an annulus $H$ which separates the two critical points 0 and $\infty$ and on which $F$ is a homeomorphism.

\section{Acknowledgement}

We would like to thank the referee for their many important suggestions which greatly improved the paper. 


\section{References}

[1] L. Carleson and T. W. Gamelin, Complex Dynamics (Springer, New York, 1993).

[2] A. Katok and B. Hasselblatt, Introduction to the Modern Theory of Dynamical Systems, Encyclopedia of Mathematics and its Applications, 54 (Cambridge University Press, New York, 1995).

[3] S. Marmi and J.-C. Yoccoz (eds.), Dynamical Systems and Small Divisors, Lecture Notes in Mathematics, 1784 (Springer, Berlin, 2002).

[4] G. Zhang, 'On the dynamics of $e^{2 \pi i \theta} \sin (z)$ ', Illinois J. Math. 49(4) (2005), 1171-1179.

XIUMEI WANG, Department of Computer Science and Information Technology, JiangSu Teachers University of Technology, Changzhou, 213001, PR China e-mail: wxmei@jstu.edu.cn

GAOFEI ZHANG, Department of Mathematics, Nanjing University, Nanjing, 210093, PR China

e-mail: zhanggf@hotmail.com 